\title{
The Comparison of Juvenile Hormone and Transcriptional Changes between Three Different Juvenile Hormone Analogs Insecticides on Honey Bee Worker Larval's Development
}

\author{
Jie Luo, Sheng Liu, Jiangan Hou, Lichao Chen, Honghong Li, Shucheng Liao, Qimei Tan, Taiyi Yang, Guoqiang Yi, \\ Fu Zhang and Xuesheng Li *
}

Citation: Luo, J.; Liu, S.; Hou, J.; Chen, L.; Li, H.; Liao, S.; Tan, Q.; Yang, T.; Yi, G.; Zhang, F.; et al. The Comparison of Juvenile Hormone and Transcriptional Changes between Three Different Juvenile Hormone Analogs Insecticides on Honey Bee Worker Larval's Development. Agronomy 2021, 11, 2497. https:// doi.org/10.3390/agronomy11122497

Academic Editors: José Carlos Franco, Arturo Cocco, Stefano Speranza,

António Onofre Costa

Miranda Soares and Lucia Zappala

Received: 1 November 2021

Accepted: 6 December 2021

Published: 9 December 2021

Publisher's Note: MDPI stays neutral with regard to jurisdictional claims in published maps and institutional affiliations.

Copyright: (c) 2021 by the authors. Licensee MDPI, Basel, Switzerland. This article is an open access article distributed under the terms and conditions of the Creative Commons Attribution (CC BY) license (https:// creativecommons.org/licenses/by/ $4.0 /)$.
Guangxi Key Laboratory of Agric-Environment and Agric-Products Safety, Guangxi University, Nanning 530004, China; roger@st.gxu.edu.cn (J.L.); $1817304009 @$ st.gxu.edu.cn (S.L.); 1917404004@st.gxu.edu.cn (J.H.); 1917304001@st.gxu.edu.cn (L.C.); 1317314001@st.gxu.edu.cn (H.L.); 2017304011@st.gxu.edu.cn (S.L.); 1917304019@st.gxu.edu.cn (Q.T.); 2017301047@st.gxu.edu.cn (T.Y.); 2017392042@st.gxu.edu.cn (G.Y.); 2117392065@st.gxu.edu.cn (F.Z.)

* Correspondence: lxsnngx@gxu.edu.cn

Abstract: Juvenile hormones (JHs) play a crucial role in the development of honey bee (Apis mellifera) worker larvae. Juvenile hormone analogs (JHAs), insecticides widely used in pest control, have been reported to affect the health and survival of honey bee worker larvae. However, the molecular mechanisms of JHAs in the honey bee remain unclear. In this study, we treated honey bee worker larvae with pyriproxyfen, fenoxycarb, and methoprene, three different JHAs. We monitored the changes in the transcription of genes encoding major JH response enzymes (CYP15A1, CYP6AS5, JHAMT, and CHT1) using RT-qPCR and analyzed the transcriptome changes in worker larvae under JHA stress using RNA-seq. We found that the enrichment pathways differed among the treatment groups, but the classification of each pathway was generally the same, and fenoxycarb affected more genes and more pathways than did the other two JHAs. Notably, treatment with different JHAs in the honey bee changed the JH titers in the insect to various extents. These results represent the first assessment of the effects of three different JHAs on honey bee larvae and provide a new perspective and molecular basis for the research of JH regulation and JHA toxicity in the honey bee.

Keywords: honey bee; juvenile hormone; pyriproxyfen; fenoxycarb; methoprene; RT-qPCR; RNA-seq

\section{Introduction}

The honey bee is widely distributed all over the world [1]. It plays a crucial role in the pollination of crops [2,3], and a healthy and strong colony of honey bees well enhances the pollination area of local crops. Thus, the honey bee greatly contributes to the social economy and ecology. The honey bee has developed into a new model organism, which, because of its extremely complex and delicate social structure and variety of behaviors, allows for the biology and behaviors of insects to be studied [4-7]. Moreover, exploring the effects of insecticides on the honey bee has always been an important component in honey bee science.

Juvenile hormones (JHs) are a group of sesquiterpenoids [8] that are indispensable endocrine hormones in insects. They are synthesized and secreted by the corpora allata $[9,10]$ and play a crucial role in the regulation of vitellogenesis, reproduction, and ovary development of insects [11-13]. They also function in insect migration, energy metabolism, and caste differentiation [14-16]. JHAs are compounds similar to JH in structure and mechanism [17-20]. JHAs can effectively mimic JHs in insects to exercise its function or interfere with JH synthesis. Consequently, changes occur in JH titers in the insect hemolymph, thereby interfering with the normal physiological and biochemical processes of insects and achieving the purpose of controlling and eliminating pests because of their similarities [21]. 
JHA insecticides include pyriproxyfen, fenoxycarb, and methoprene. Previous research has shown that pyriproxyfen can reduce the growth rate of Hippodamia convergens and inhibit their population. Fenoxycarb reduces the longevity of pear psylla (Cacopsylla pyricola). Methoprene is an important mediator of female Drosophila melanogaster, which, when treated with methoprene, exhibits sexual inhibition to male $D$. melanogaster after mating [22-24]. Other studies have suggested that JHA insecticides can affect the social activity of the honey bee and change its growth and development processes $[25,26]$. Genes such as cytochrome CYP6AS5 (CYP6AS5), chitinase (CHT1), cytochrome CYP15A1 (CYP15A1), and juvenile hormone acid methyltransferase (JHAMT) reportedly induce responses when insects are under JHA stress [27-29]. Currently, with the wider use of JHA insecticides in pest control, it is becoming increasingly important to test the toxicity of pyriproxyfen, fenoxycarb, and methoprene in honey bees as well as their regulation and molecular mechanisms.

In the present study, changes in the JH titers inside the honeybees' hemolymphs were measured after treatment with three different JHAs. We also present an analysis of the treatment transcriptome, for which high-throughput RNA-seq technology was utilized to identify and quantify the expression levels of the genes transcribed in honey bees' larvae, which had been exposed to three different JHAs. The genes related to metabolism processes and physiological changes were then identified and analyzed. Moreover, the differentially expressed genes (DEGs) possibly involved in the physiological metabolism and detoxification responses were identified and verified using the RT-qPCR method. The resulting transcriptome library may help to elucidate the regulation and molecular mechanisms of JHA on honey bees and screen the most effective JHA pesticide among these three JHAs in honey bees.

\section{Materials and Methods}

\subsection{Chemicals and Honey Bee Larvae Rearing}

Pyriproxyfen, fenoxycarb, methoprene standard, JH III, HPLC-grade methanol, acetonitrile, and isooctane were purchased from ANPEL Laboratory Technologies (Shanghai) Inc. Cell culture plates, D-glucose, D-fructose, yeast extract, and other reagents (analytical reagents) were obtained from Sangon Bioengineering (Shanghai Co., Ltd., Shanghai China). Royal jelly was supplied by a bee keeper in Nanning.

Our experiments were conducted during the winter of 2020 at the GuangXi University Insect Application Laboratory $\left(22^{\circ} 50^{\prime} \mathrm{N}, 108^{\circ} 17^{\prime} \mathrm{E}\right)$, Nanning Guangxi, China. All honey bee larvae samples were collected from $A$. mellifera ligustica colonies. Three treatment groups and one control group were established, and three different JHAs were used separately in different treatment groups.

In order to obtain precisely aged larvae, a queen was captured and locked in an empty comb for $4 \mathrm{~h}$ for egg laying. Newly emerged larvae $(<12 \mathrm{~h}$ old $)$ were transferred to a clean 48 -well cell culture plates, which were placed in sterile queen cell cups containing $25 \mu \mathrm{L}$ of $\operatorname{diet} \mathrm{A}$, as shown in Table 1 . All plates were placed in an incubator $\left(34.5^{\circ} \mathrm{C}, 95 \%\right.$ Relative Humidity), and the day of larval removal was defined as day 1 ; no diet was served on day 2. A total of $25 \mu \mathrm{L}$ of diet B was provided on day 3, and 30 and $40 \mu \mathrm{L}$ of diet $\mathrm{C}$ were provided on days 4 and 5, respectively. All our rearing methods were conducted following the standard beekeeping methods for breeding [30].

Table 1. Diets used for larvae rearing.

\begin{tabular}{cccc}
\hline Component & Diet A (Day1) & Diet B (Day3) & Diet C (Day4, 5) \\
\hline Royal jelly & $50 \%$ & $50 \%$ & $50 \%$ \\
Glucose & $6 \%$ & $7.5 \%$ & $9 \%$ \\
Fructose & $6 \%$ & $7.5 \%$ & $9 \%$ \\
Yeast Extract & $1 \%$ & $1.5 \%$ & $2 \%$ \\
$\mathrm{H}_{2} \mathrm{O}$ & $37 \%$ & $33.5 \%$ & $30 \%$ \\
\hline
\end{tabular}


The concentration of each JHA for treatment was previously described by Tasei and Milchreit [31,32]. In this report, the maximum fenoxycarb concentration found in fieldrealistic doses ranged between 7.5 and $217 \mathrm{ng} / \mu \mathrm{L}$. Our former research (not published) shows that most honey bee larvae will not survive when they are exposed to $100 \mathrm{ng} / \mu \mathrm{L}$ of these three JHAs. Here, we chose $50 \mathrm{ng} / \mu \mathrm{L}$ for each JHA (dissolved in 0.1\% $v / v$ DMSO) [32]. Generally, different treatments and times may lead to discrepant conclusions, even when experimental subjects are exposed to identical concentrations in toxicology tests. Thus, a necessary pre-experiment was conducted to demonstrate that there was no apparent difference in mortality between control and $50 \mathrm{ng} / \mu \mathrm{L}$ of each JHA-treated group. According to the sequence of pyriproxyfen, fenoxycarb, methoprene, their $p$-values $=0.230,0.422$, and 0.768 , respectively, but all of their adjusted $p>0.05$ (independent sample $t$-test) (Table S1). Hence, it was considered suitable to apply a $50 \mathrm{ng} / \mu \mathrm{L}$ concentration of these three JHAs to honey bees in our experiments. We used $1 \mu \mathrm{L}$ of different JHAs (dissolved in $0.1 \%$ $v / v$ DMSO) and added them directly to diet $C$ at a concentration of $50 \mathrm{ng} / \mu \mathrm{L}$ on day 4 (50 ng/bee); the control group was provided with a total of $1 \mu \mathrm{L} \mathrm{DMSO}(0.1 \% v / v)$ without any JHAs in diet C, and only healthy six-day-old ( $48 \mathrm{~h}$ after day 4 ) larvae (raised using our method and no food left in the plate) were used for future tests. Three biological replicates were prepared for each group. All six-day-old bee larva samples were used for the extraction of JH, RT-qPCR experiments, and transcriptome analysis.

\subsection{Extraction and Determination of JH Titers in Honey Bees}

JH III is known as the only hormone found in the worker honey bee [33]. Herein, the method used to extract JH III was based on the method described by Furuta [34]. Six-day-old honey bee samples weighing $1 \mathrm{~g}$ (about 11 6-day-old larvae; the actual weight of each repeat was recorded for later JH III level calculations) were anesthetized at a low temperature, placed in a grinding pestle, and cooled by liquid nitrogen. After adding liquid nitrogen, the honey bees were continuously ground into powder by automatic grinding. The bee powder was transferred into a $10 \mathrm{~mL}$ centrifuge tube, and then $0.2 \mathrm{~mL}$ of acetonitrile and $1 \mathrm{~mL}$ of methanol were added after grinding was completed. The sample was mixed in a shaker for $3 \mathrm{~min}$ to which $2 \mathrm{~mL}$ of isooctane was added, and it was shaken again for another $3 \mathrm{~min}$ for complete mixing. Centrifugation was performed at $4000 \mathrm{rpm}$ for $5 \mathrm{~min}$, after which the supernatant was carefully poured into a new centrifuge tube. The isooctane extraction and supernatant absorption steps were repeated twice. Finally, a supernatant of about $6 \mathrm{~mL}$ was dried with nitrogen-blowing apparatus. After drying, $1 \mathrm{~mL}$ of acetonitrile (HPLC-grade) was added to the centrifuge tube, and the solution was passed through a green organic membrane (purification equipment). Finally, $0.2 \mathrm{~mL}$ of solution was poured into a small sample bottle for future determination.

Agilent 1260 series high-performance liquid chromatography-tandem mass spectrometry (Agilent Technologies, Inc., Colorado Springs, CO, USA) was used for chromatographic analysis. The chromatographic column was CHIRALPAK AY-3R $(150 \mathrm{~mm} \times 2.1 \mathrm{~mm}, 3 \mu \mathrm{m}$; Daicel Chiral Technologies, Shanghai, China). The column temperature was $30{ }^{\circ} \mathrm{C}$, the mobile phase was $0.1 \%$ formic acid and acetonitrile ( $\mathrm{v}: \mathrm{v}=55: 45)$, the flow rate was $0.3 \mathrm{~mL} / \mathrm{min}$, the injection volume was $3 \mu \mathrm{L}$, the mobile phase was $0.1 \%$ formic acid water-acetonitrile solution, and the detection wavelength was $214 \mathrm{~nm}$. The mass spectrometry conditions were as follows: electrospray ion source and positive ion scanning mode (ESI+); capillary voltage, $4.0 \mathrm{kv}$; air temperature, $330{ }^{\circ} \mathrm{C}$; and atomizing gas pressure, $103.425 \mathrm{kPa}$. Multiple response monitoring mode was used with isocratic elution. Qualitative analysis was carried out based on retention time and ion information comparison. The parent ion and ion with the highest response value were quantitatively analyzed for the R-JH III and S-JH III retention time, ion monitoring, pyrolysis voltage, and collision energy.

\subsection{RNA Extraction, Library Preparation, and Sequencing}

The total RNA of honey bee larvae was extracted with a Trizol (MRC Cincinnati, $\mathrm{OH}, \mathrm{USA}$ ) reagent kit according to the manufacturer's instructions. The biological repli- 
cates of pyriproxyfen, fenoxycarb, and methoprene treatment groups were pyriproxyfen-1, pyriproxyfen-2, and pyriproxyfen-3; fenoxycarb-1, fenoxycarb-2, and fenoxycarb-3; and methoprene-1, methoprene-2, and methoprene-3, respectively. The biological replicates of the control group were control-1 (CK-1), control-2 (CK-2), and control-3 (CK-3). For all groups, three specimens were collected for each sample, three replicates were performed, and the quantity and quality of RNA were assessed using a NanoDrop 2000 spectrophotometer (Thermo Fisher Scientific). The used reverse transcription kit was Hifair ${ }^{\circledR}$ III 1st Stand cDNA Synthesis Supermix for qPCR (gDNA digester plus) (Yeasen Biotechnology (Shanghai) Co., Ltd., Shanghai, China). Quantitative real-time PCR was carried out using a QuantStudio 6 Flex instrument (Thermo Fisher Scientific) at $95{ }^{\circ} \mathrm{C}$ for $30 \mathrm{~s}$ for pre-denaturation and 40 cycles of $95^{\circ} \mathrm{C}$ for $5 \mathrm{~s}$ and $60^{\circ} \mathrm{C}$ for $30 \mathrm{~s}$ for PCR. The total reaction volume was $10 \mathrm{~mL}$. Primer sequences for genes encoding JHA stress enzymes and internal controls were designed using Primer-BLAST (NCBI). RNA samples were prepared for a subsequent study.

RNA samples were collected for cDNA library construction. Agilent 2100 Bioanalyzer (Agilent Technologies, Santa Clara, CA, USA) and Qubit fluorometer (Invitrogen, Carlsbad, CA, USA) were used to determine the RNA quantity and quality, respectively. Three replicates each of the control and treatment groups were analyzed. Total RNA extraction steps were the same. Library preparation and whole-transcriptome sequencing were performed on the Illumina sequencing platform developed by Genedenovo Biotechnology Co., Ltd. (Guangzhou, China). RNA integrity was checked using standard agarose gel electrophoresis with TAE buffer. First-strand cDNA was synthesized in the M-MuLV reverse transcriptase system with fragmentized mRNA as a template and random oligonucleotides as primers. The RNA strand was then degraded with RNaseH. Second-strand cDNA was synthesized from dNTPs in the DNA polymerase I system. Random hexamer primers were used for library preparation and amplification. The AMPure XP System (Beckman Coulter, Brea, CA, USA), was used to purify double-stranded cDNA, which was then eluted with EB buffer for end repair and the addition of poly (A). Illumina HiSeqTM2000 (Illumina, San Diego, CA, USA) was used to sequence the cDNA library and generate about $200 \mathrm{bp}$ paired-end reads.

\subsection{Transcriptomic Data Analysis}

Raw data (raw reads) in FASTA format were processed through in-house perl scripts (NCBI number: PRJNA734139). In this step, clean data (clean reads) were obtained by removing reads containing an adapter, reads with all A bases, reads containing ploy- $\mathrm{N}$, and low-quality reads from raw data. Meanwhile, the contents of GC, Q20, and Q30 of clean data were calculated. All downstream analyses were based on high-quality clean data and HISAT2 was used to count the read numbers mapped to each gene. The FPKM of each gene was then calculated based on the gene length and read count mapped to this gene.

\subsection{Functional Analyses of DEGs}

The relative expression levels of genes among the control, pyriproxyfen-treated, fenoxycarb-treated, and methoprene-treated groups were analyzed using the DEGseq method [35], which provided statistical routines to determine differential expression in digital gene expression data by using a model based on the negative binomial distribution. Corrected $p$-value $<0.05$ and $\log 2$ (fold change) $\geq 1$ were set as the threshold for significantly differential expression. GO terms with corrected $p$-values less than 0.05 were considered as significantly enriched by DEGs. KOBAS software was used to determine the statistical enrichment of DEGs in Kyoto Encyclopedia of Genes and Genomes (KEGG) pathways.

\subsection{Validation of Four DEGs}

Four candidate differentially regulated JHA-sensitive genes with various biological functions were analyzed by RT-qPCR by using three biological replicates to verify the transcriptome results. We selected four DEGs related to JHA stress, CHT1, CYP6AS5, 
CYP15A1, and JHAMT genes, which were expressed in three different treatments (Table S2). About $10 \mu \mathrm{L}$ of PCR reaction volume contained $5 \mu \mathrm{L}$ of TB Green TM Pre mix Ex taqTMII, $3 \mu \mathrm{L}$ of DEPC water, $1 \mu \mathrm{L}$ of diluted cDNA template, and $0.5 \mu \mathrm{L}$ of each primer. The PCR reaction was performed on the Step One Plus TM Real-Time System (Bio Rad, USA) under the following conditions: $95^{\circ} \mathrm{C}$ for $30 \mathrm{~s}$ for the holding stage; 40 cycles of $95{ }^{\circ} \mathrm{C}$ for $5 \mathrm{~s}$, $60{ }^{\circ} \mathrm{C}$ for $34 \mathrm{~s}$ for the PCR stage. A final melting curve analysis was performed. The relative expression levels of the selected transcripts normalized to the housekeeping gene (actin) were calculated using the $2^{-\Delta \Delta C T}$ method [36]. $\Delta \Delta \mathrm{Ct}=(\mathrm{Ct}$, Target $-\mathrm{Ct}$, Actin $)$ treat $-(\mathrm{Ct}$, Target $-\mathrm{Ct}$, Actin) control.

\subsection{Statistics}

Student $t$-tests were used to determine the significance of DEGs expression differences between control and treatment groups (RNA-seq and RT-qPCR). Two-tailed probabilities were adopted in the tests. Multistrata parametric tests were used to determine the significance of JH III titer changes between control and treatment groups. All analyses were performed with SPSS Statistics 21.0 software (SPSS Inc., Chicago, IL, USA).

\section{Result}

\subsection{Determination of JH Titers in Six-Day-Old Worker Larvae}

The JH content in honey bee larvae after different JHA treatments was determined by LC-MS. Three kinds of JHA changed the JH titers in honey bee larvae. Among them, the $\mathrm{JH}$ titers in honey bee larvae treated with fenoxycarb were the lowest. The JH titers treated with methoprene were slightly higher than those treated with fenoxycarb. The JH titers treated with pyriproxyfen were the highest (Figure 1).

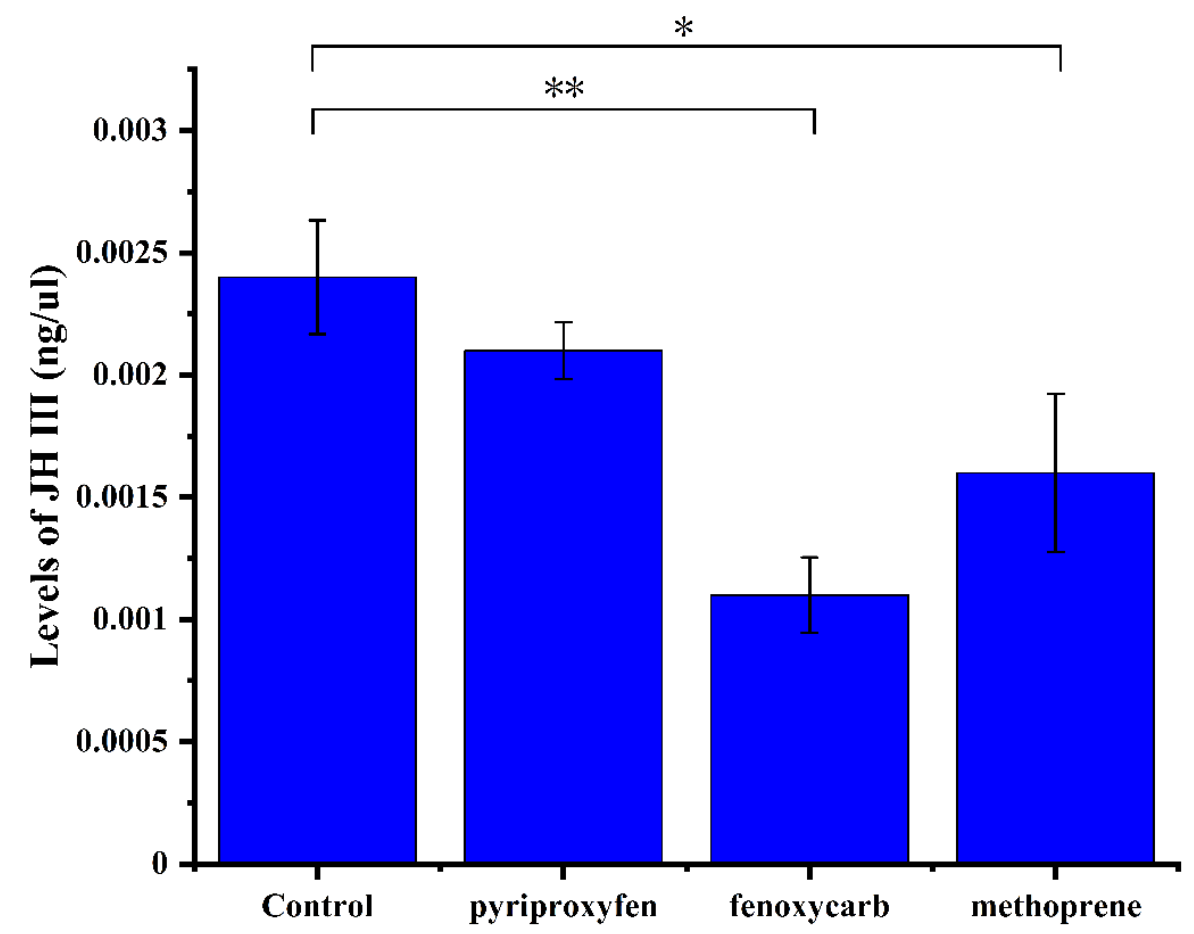

Figure 1. Titers of JH III in 6-days-old larvae after different JHAs exposure and in the control group. Data presented in the table are mean \pm standard error. $X$-axis represents the control group and different-treated groups. Y-axis shows the titers level of JH III. With significant differences between different treatment groups and control groups are indicated using asterisks: ${ }^{*} p<0.05,{ }^{* *} p<0.01$ (post-hoc parametric test) (Table S3). 


\subsection{Quality Analysis of Transcriptome Sequencing Results}

After filtering low-quality data, clean reads were obtained. The effective reads of RNA-seq ranged from $40,097,124$ to $58,162,002$, with the lowest unique matching rate of $85.10 \%$ and the highest $88.98 \%$. Q30 values were also above $90.14 \%$ (Table S4). This finding showed that the quality of the RNA-seq data was high. The final sequencing results were reliable.

\subsection{Screening of DEGs}

Based on the results of differential analyses, we screened the false discovery rate (FDR) $<0.05$ and $\log 2 \mathrm{FC}>1$ as a DEGs. A total of 34, 532, and 222 DEGs (Table S5) were identified among the three different JHA treatment and control groups. Among them, 21 were upregulated and 13 were downregulated in the pyriproxyfen-treated group compared with the control group; 222 were upregulated and 310 were downregulated in the fenoxycarb-treated group compared with the control group; and 155 were upregulated and 67 were downregulated in the methoprene-treated group compared with the control group (Figure 2A). Moreover, the results for the DEGs as visualized via a Venn diagram (Figure 2B) revealed that 34,532 , and 222 genes were specifically expressed in the different JHA treatment groups, and they had two DEGs in common.

A

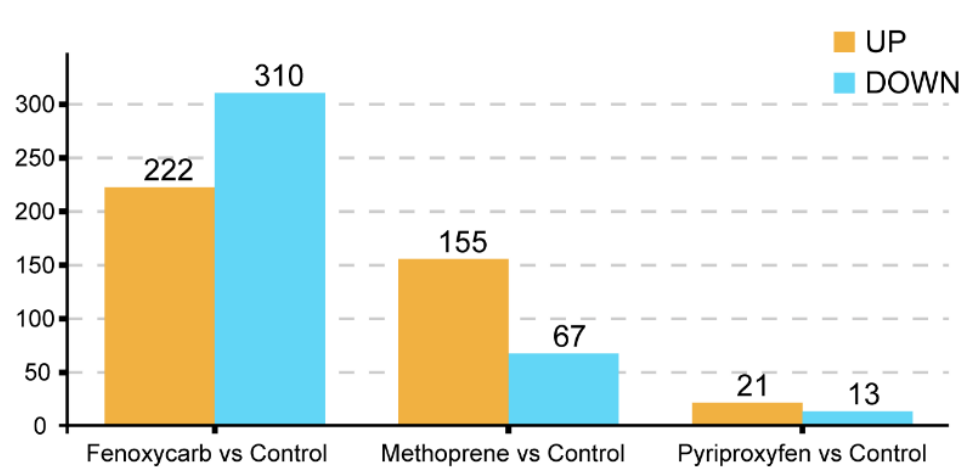

B

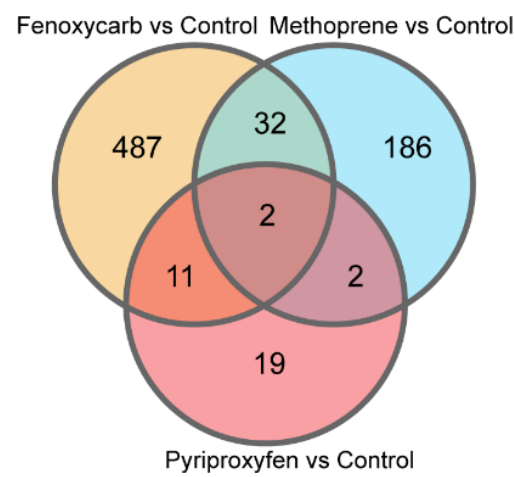

Figure 2. Histogram of DEGs between different JHA treated and control groups. (A) The number of DEGs induced by the $\mathrm{X}$-axis indicates the name of each groups; Y-axis shows the gene number of each different groups. (B) Venn diagram of shared DEGs between each groups.

\subsection{GO Functional Annotation and KEGG Pathway}

GO annotations were classified into the category of biological processes, molecular functions, and cellular components. When the $p$-value was $<0.05$, it was considered as an enrichment item. Among the DEGs in the pyriproxyfen-treated group, 91 genes with GO annotation were enriched to $20 \mathrm{GO}$ terms; 1567 gens with GO annotation were enriched to $38 \mathrm{GO}$ terms in the fenoxycarb-treated group; and 796 genes with GO annotation were enriched to $33 \mathrm{GO}$ terms in the methoprene-treated group. The GO annotations for the DEGs of different JHA treatment groups are shown in Figure S1. The top 20 significantly enriched GO terms of each JHA treatment group are shown in Figure S2.

The biochemical pathways of the DEGs were investigated using the KEGG database. KEGG analysis suggested that 49 DEGs were divided into 31 pathways in the pyriproxyfentreated group. In the fenoxycarb-treated group, a total of 601 DEGs were assigned to 227 pathways. In the methoprene-treated group, 409 DEGs were involved in 171 pathways. The top 20 significantly enriched KEGG pathways are shown in Figure S3.

\subsection{Validation of DEGs by Using RT-qPCR}

To verify the reliability of the RNA-Seq data, four DEGs involved in the JH response were selected and verified using the RT-qPCR method. The results showed a similar trend 
of expression changes for RT-qPCR and RNA-Seq data, indicating that the RNA-Seq results were reliable (Figure 3), and the FPKM mean value of RNA-seq and RT-qPCR data had been shown in Tables 2 and 3.

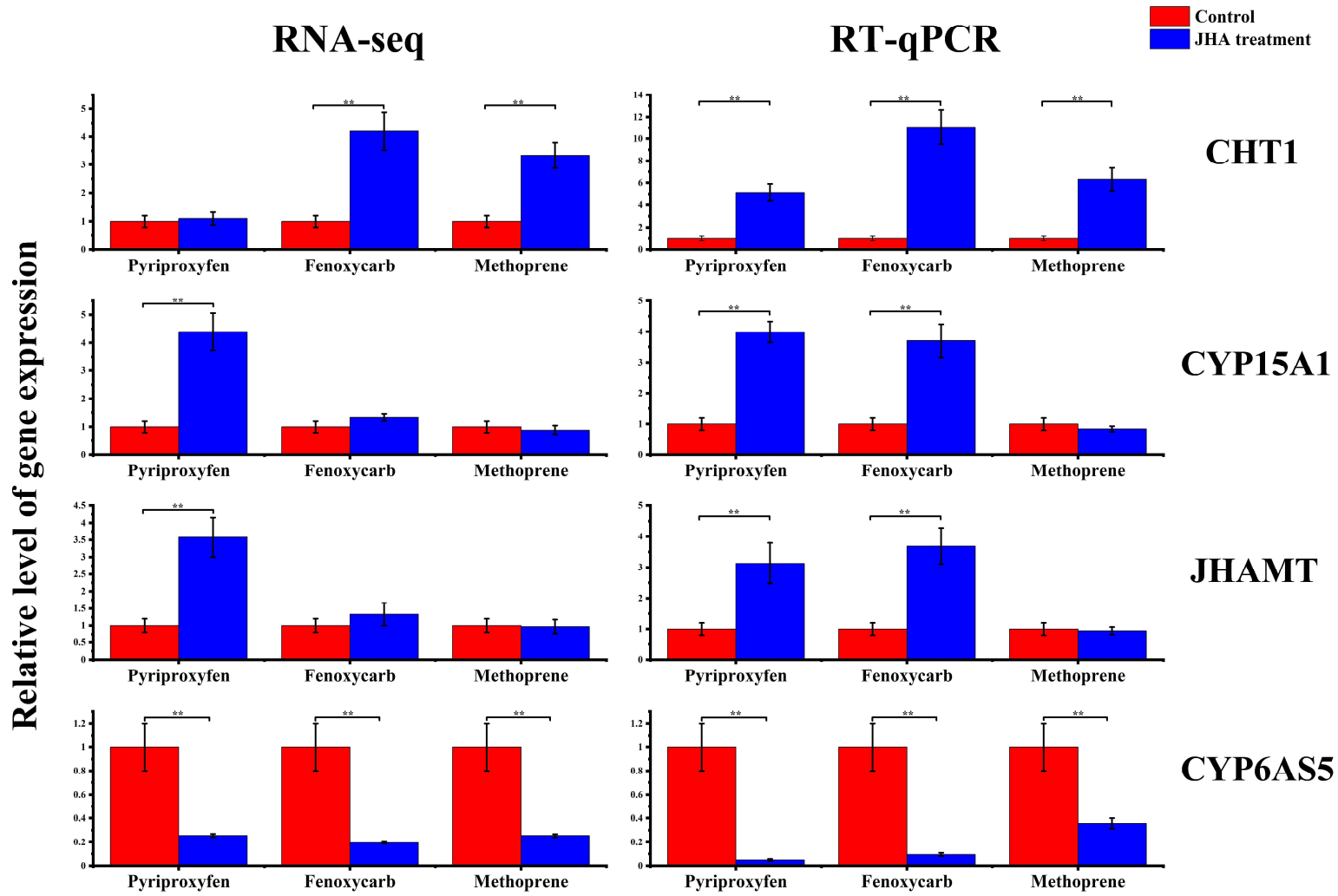

Joint analysis of RNA-seq (Left) and qRT-PCR (Right) of four DEGs in different treatments

Figure 3. Confirmation of RNA-Seq results by RT-qPCR. Data presented in the figure are mean \pm standard error. X-axis represents different treatment groups. Y-axis shows the relative level of gene expression. With significantly expression differences between different treatment groups and control groups are indicated using asterisks: ${ }^{* *} p<0.01$ (independentsample $t$-test).

Table 2. The FPKM mean value of RNA-seq of different genes in different treatment groups.

\begin{tabular}{cccc}
\hline Gene & Pyriproxyfen & Fenoxycarb & Methoprene \\
\hline CHT1 & 1.10 & 4.20 & 3.34 \\
CYP6AS5 & 0.26 & 0.19 & 0.25 \\
CYP15A1 & 4.40 & 1.33 & 0.88 \\
JHAMT & 3.58 & 1.33 & 0.97 \\
\hline
\end{tabular}

Table 3. The mean value of RT-qPCR of different genes in different treatment groups.

\begin{tabular}{cccc}
\hline Gene & Pyriproxyfen & Fenoxycarb & Methoprene \\
\hline CHT1 & 5.13 & 11.08 & 6.31 \\
CYP6AS5 & 0.05 & 0.09 & 0.36 \\
CYP15A1 & 3.99 & 3.70 & 0.84 \\
JHAMT & 3.13 & 3.69 & 0.94 \\
\hline
\end{tabular}

\section{Discussion}

JHs are important hormones in honey bees, as they regulate the growth and development of honey bee larvae. JHs can increase food consumption and reduce fat amounts in worker and queen bumble bees (Bombus terrestris) [37]. Former researchers discovered 
that there are JH titer differences between honey bee workers and queens [38,39], and they also proved that JHs are indispensable hormones in the developmental determination of queen-like characters $[40,41]$. In our study, the application of JHAs at environmental concentrations affected the JH titers of honey bees. Bee larvae with such hormonal changes probably fail to molt or become abnormal workers or queens. In addition, we do not know how long the negative effects of this elevated JH level will last and whether it will continue to affect bees after metamorphosis; thus, more work studying the effects of JH III abnormalities induced by JHAs in the larva stage on the life cycle of adults is necessary.

The synthesis and metabolism of JHs in insects are well understood, but the mechanism regulating their levels remains unclear. In our experiment, when exposure to different JHAs downregulated JH III levels, the RT-qPCR result of all related metabolic enzymes showed the same expression patterns as those of the transcriptome results, and the RT-qPCR results of some genes showed significant changes when compared with their transcriptome results in different treatments. Our results indicate that the expressions of CYP15A1 and JHAMT in different treatments were upregulated. As important enzymes related to JH metabolism [41-43], CYP15A1 and JHAMT should increase JH titers in insects when they are upregulated, but our results suggest that the JH titers show downregulation when CYP15A1 and JHAMT are upregulated. This may be related to the expression of other metabolic enzymes of JHs, such as juvenile hormone esterase and juvenile hormone epoxide hydrolase (Table S6). In addition, hormone regulation in an insect is complex and involves a large number of factors. Ion levels, insulin signaling [44], and allatostatins [9,45] are also considered influencing factors for JH synthesis.

Cytochrome P450 enzymes are well-known for their metabolism of pesticides [46,47], not only CYP15A1 as discussed above but also the CYP6AS5. In our experiment, we demonstrated that the CYP6AS subfamily is unique to hymenopterans. Wang et al. [48] showed that carbendazim decreases $\mathrm{JH}$ titer levels in honey bee larvae, accompanied by the downregulation of CYP6AS5, which shares the same trend with our results. A previous study conducted by our group demonstrated that CYP6AS5 is upregulated when honey bees are exposed to thiamethoxam. In addition, JH levels show an increasing trend [49]. Thus, a positive correlation between the level of JHs in insect larvae and CYP6AS5 transcription in honey bees was observed. We speculate that CYP6AS5 is consistent with CYP15A1 and CYP4C7, and that it plays an important role in regulating JH titers [50,51].

There are large differences in the number of DEGs in different treatment groups. Figures S2 and S3 indicate that the top 20 significantly enriched GO terms and KEGG pathways were different among the different treatments. This result shows that the effects of the molecular mechanisms of pyriproxyfen, fenoxycarb, and methoprene on honey bees are different, which may explain the results of JH titers. Figure S1 shows that fenoxycarb affected more genes and more pathways than did the other two JHAs, and it also showed a stronger effect on the control JH titers.

In conclusion, the results of this study show that the environmental concentration of these three JHAs significantly reduced the level of JH III in honey bee worker larvae, which may harm honey bee caste differentiation. The data of our RNA-seq results reveal that these JHAs affected the catalytic activity, biosynthetic process, and metabolism pathways. We speculate that some of these alternations are related to the regulation of $\mathrm{JH}$ levels. Our results provide a new perspective and molecular basis for the study of the effect of JHA mechanisms on honey bees.

Supplementary Materials: The following are available online at https:/ / www.mdpi.com/article/10 .3390 /agronomy11122497/s1. Figure S1: Correlation coefficient of samples, a histogram representation of the GO terms in different treatment groups. Figure S2: Top 20 significantly enriched GO terms of different treatments. Figure S3: Top 20 significantly enriched KEGG pathways of different treatments. Table S1: Survival rate. Survival rates of control and $50 \mathrm{ng} / \mathrm{ul}$ of each treatment groups (t-Tests). Table S2: Gene-specific primers. Specific primers of functional gene used for RT-qPCR. Table S3: The post-hoc parametric test of the effect of different JHA treatment on JH III titer changes. Table S4: Summary of mapping result of RNA-seq. All genome mapping statistics. Table S5: Top 20 
DEGs of each treatment groups. Table S6: The FPKM mean value of RNA-seq of different JHE and JHEH in different treatment groups.

Author Contributions: Writing—review and editing \& writing-original draft preparation, J.L.; Methodology, S.L. (Sheng Liu); Supervision, J.H.; Software, L.C. and F.Z.; Data curation, H.L. and Q.T.; Resources, S.L. (Shucheng Liao) and G.Y.; Visualization, T.Y.; Funding acquisition and Investigation, X.L. All authors have read and agreed to the published version of the manuscript.

Funding: This work was supported by Key Research and Development Program of Guangxi (Guike AA17204043).

Data Availability Statement: All transcription data were uploaded to the NCBI SRA database (PRJNA734139).

Acknowledgments: We Thank Guoqiang Xu and Gene Denovo Biotechnology Co., Ltd. (Guangzhou, China) helping with transcriptome sequencing and valuable suggestions.

Conflicts of Interest: The authors declare that there is no competing interest and that the article is submitted without any commercial or economic interest that could be generated as a potential conflict of interest.

\begin{tabular}{ll}
\multicolumn{2}{l}{ Abbreviations } \\
JH & Juvenile hormone \\
JHA & Juvenile hormone analog \\
CHT1 & Chitinase \\
CYP15A1 & Cytochrome CYP15A1 \\
CYP6AS5 & Cytochrome CYP6AS5 \\
JHAMT & Juvenile hormone acid methyltransferase \\
FPKM & Fragments Per Kilobase per Million \\
DMSO & Dimethyl sulfoxide \\
CK & Control check \\
Bbm & Pyriproxyfen \\
Byw & Fenoxycarb \\
Xcz & Methoprene
\end{tabular}

\section{References}

1. Ikeda, T.; Furukawa, S.; Nakamura, J.; Sasaki, M.; Sasaki, T. CpG methylation in the hexamerin 110 gene in the European honeybee, Apis mellifera. Insect Sci. 2011, 11, 74. [CrossRef] [PubMed]

2. Sillman, J.; Uusitalo, V.; Tapanen, T.; Salonen, A.; Soukka, R.; Kahiluoto, H. Contribution of honeybees towards the net environmental benefits of food. Sci. Total Environ. 2021, 756, 143880. [CrossRef]

3. Nakamura, S.; Yamamoto, S.; Sawamura, N.; Nikkeshi, A.; Kishi, S.; Kamo, T. Pollination effectiveness of European honeybee, Apis mellifera (Hymenoptera: Apidae), in an Oriental persimmon, Diospyros kaki (Ericales: Ebenaceae), orchard. Appl. Entomol. Zool. 2020, 55, 405-412. [CrossRef]

4. Dearden, P.K.; Duncan, E.J.; Wilson, M.J. The honeybee Apis mellifera. Cold Spring Harb. Protoc. 2009, 2009, emo123. [CrossRef]

5. Wang, X.; Zhang, X.; Zhang, Z.; Lang, H.; Zheng, H. Honey bee as a model organism to study gut microbiota and diseases. Drug Discov. Today Dis. Models. 2018, 28, 35-42. [CrossRef]

6. Kohno, H.; Kubo, T. Genetics in the Honey Bee: Achievements and Prospects toward the Functional Analysis of Molecular and Neural Mechanisms Underlying Social Behaviors. Insects 2019, 10, 348. [CrossRef]

7. Richards, M.H. Socially polymorphic bees as model organisms for studying the evolution of eusociality. Insectes Sociaux. 2019, 66, 3-4. [CrossRef]

8. Kelstrup, H.C.; Hartfelder, K.; Nascimento, F.S.; Riddiford, L.M. The role of juvenile hormone in dominance behavior, reproduction and cuticular pheromone signaling in the caste-flexible epiponine wasp, Synoeca surinama. Front. Zool. 2014, 11, 78. [CrossRef]

9. Stay, B.; Tobe, S.S. The role of allatostatins in juvenile hormone synthesis in insects and crustaceans. Annu. Rev. Entomol. 2007, 52, 277-299. [CrossRef]

10. Denlinger, D.L.; Yocum, G.D.; Rinehart, J.P. Hormonal Control of Diapause. Endocrinology 2012, 430-463. [CrossRef]

11. Wyatt, G.R.; Davey, K.G. Cellular and molecular actions of juvenile hormone. II. Roles of juvenile hormone in adult insects. Adv. Insect Physiol. 1996, 6, 1-155. [CrossRef]

12. Fei, H.; Martin, T.R.; Jaskowiak, K.M.; Hatle, J.D.; Whitman, D.W.; Borst, D.W. Starvation affects vitellogenin production but not vitellogenin mRNA levels in the lubber grasshopper, Romalea microptera. J. Insect Physiol. 2005, 51, 435-443. [CrossRef] 
13. Xiao, H.J.; Fu, X.W.; Liu, Y.Q.; Wu, K.M. Synchronous vitellogenin expression and sexual maturation during migration are negatively correlated with juvenile hormone levels in Mythimna separata. Sci. Rep. 2016, 6, 33309. [CrossRef]

14. Wang, Y.; Brent, C.S.; Fennern, E.; Amdam, G.V. Gustatory perception and fat body energy metabolism are jointly affected by vitellogenin and juvenile hormone in honey bees. PLoS Genet. 2012, 8, e1002779. [CrossRef]

15. Zhang, L.; Cheng, L.; Chapman, J.W.; Sappington, T.W.; Liu, J.; Cheng, Y.; Jiang, X. Juvenile hormone regulates the shift from migrants to residents in adult oriental armyworm, Mythimna separata. Sci. Rep. 2020, 10, 11626. [CrossRef] [PubMed]

16. Araki, M.; Miyakawa, M.O.; Suzuki, T.; Miyakawa, H. Two insulin-like peptides may regulate egg production in opposite directions via juvenile hormone signaling in the queenless ant Pristomyrmex punctatus. J. Exp. Zool. Part B Mol. Dev. Evol. 2020, 334, 225-234. [CrossRef] [PubMed]

17. Wilson, T.G. The molecular site of action of juvenile hormone and juvenile hormone insecticides during metamorphosis: How these compounds kill insects. J. Insect. Physiol. 2004, 50, 111-121. [CrossRef]

18. Saltzmann, K.A.; Saltzmann, K.D.; Neal, J.J.; Scharf, M.E.; Bennett, G.W.J. Effects of the juvenile hormone analog pyriproxyfen on German cockroach, Blattella germanica (L.), tergal gland development and production of tergal gland secretion proteins. Arch. Insect Biochem. Physiol. 2010, 63, 15-23. [CrossRef]

19. Cripe, G.M.; Mckenney, C.L.; Hoglund, M.D.; Harris, P.S. Effects of fenoxycarb exposure on complete larval development of the xanthid crab, Rhithropanopeus harrisii. Environ. Pollut. 2003, 125, 295-299. [CrossRef]

20. Hu, X.L.; Niu, J.J.; Meng, Q.; Chai, Y.H.; Chu, K.H.; Chan, K.M. Effects of two juvenile hormone analogue insecticides, fenoxycarb and methoprene, on Neocaridina davidi. Environ. Pollut. 2019, 253, 89-99. [CrossRef]

21. Hu, X.L.; Tang, Y.Y.; Kwok, M.L.; Chan, K.M.; Chu, K.H. Impact of juvenile hormone analogue insecticides on the water flea Moina macrocopa: Growth, reproduction and transgenerational effect. Aquat. Toxicol. 2020, 220, 105402. [CrossRef] [PubMed]

22. Iftikhar, A.; Hafeez, F.; Hafeez, M.; Farooq, M.; Asif Aziz, M.; Sohaib, M.; Naeem, A.; Lu, Y. Sublethal effects of a juvenile hormone analog, Pyriproxyfen on demographic parameters of non-target predator, Hippodamia convergens Guerin-Meneville (Coleoptera: Coccinellidae). Ecotoxicology 2020, 29, 1017-1028. [CrossRef]

23. Horton, D.R.; Lewis, T.M.J. Effects of fenoxycarb on ovarian development, spring fecundity and longevity in winterform pear psylla. Entomologia Experimentalis Applicata. 2015, 81, 181-187. [CrossRef]

24. Adnan, S.M.; Farhana, I.; Rempoulakis, P.; Taylor, P.W. Methoprene-induced matings of young Queensland fruit fly males are effective at inducing sexual inhibition in females. J. Appl. Entomol. 2020, 144, 500-508. [CrossRef]

25. Boleli, I.C.; Bitondi, M.; Figueiredo, V.; Simões, Z. Mispatterning in the ommatidia of Apis mellifera pupae treated with a juvenile hormone analogue. J. Morphol. 2010, 249, 89-99. [CrossRef]

26. Harano, K.I. Effects of juvenile hormone analog on physiological and behavioral maturation in honeybee drones. Apidologie 2013, 44, 586-599. [CrossRef]

27. Fujita, K.; Shimomura, K.; Yamamoto, K.; Yamashita, T.; Suzuki, K. A chitinase structurally related to the glycoside hydrolase family 48 is indispensable for the hormonally induced diapause termination in a beetle. Biochem. Biophys. Res. Commun. 2006, 345, 502-507. [CrossRef] [PubMed]

28. Helvig, C.; Koener, J.F.; Unnithan, G.C.; Feyereisen, R. CYP15A1, the cytochrome P450 that catalyzes epoxidation of methyl farnesoate to juvenile hormone III in cockroach corpora allata. Proc. Natl. Acad. Sci. USA 2004, 101, 4024-4029. [CrossRef] [PubMed]

29. Luo, W.; Veeran, S.; Wang, J.; Li, S.; Li, K.; Liu, S.N. Dual roles of juvenile hormone signaling during early oogenesis in Drosophila. Insect Sci. 2020, 27, 665-674. [CrossRef]

30. Tavares, D.A.; Dussaubat, C.; Kretzschmar, A.; Carvalho, S.M.; Silva-Zacarin, E.C.M.; Malaspina, O.; Berail, G.; Brunet, J.L.; Belzunces, L.P. Exposure of larvae to thiamethoxam affects the survival and physiology of the honey bee at post-embryonic stages. Environ. Pollut. 2017, 229, 386-393. [CrossRef]

31. Tasei, J. Impact of agrochemicals on non-Apis bees. In Honey Bees: Estimating the Environmental Impact of Chemicals; CRC Press: Boca Raton, FL, USA, 2002; ISBN 978-0-415-27518-7.

32. Milchreit, K.; Ruhnke, H.; Wegener, J.; Bienefeld, K.J.E. Effects of an insect growth regulator and a solvent on honeybee (Apis mellifera L.) brood development and queen viability. Ecotoxicology 2016, 25, 530-537. [CrossRef] [PubMed]

33. Sasagawa, H.; Kuwahara, Y. Quantitative Determination Method for a Juvenile Hormone III Titer in Honeybee Haemolymph by High-Performance Liquid Chromatography. Agric. Biol. Chem. 2016, 52, 1295-1297. [CrossRef]

34. Furuta, K.; Ichikawa, A.; Murata, M.; Kuwano, E.; Shiotsuki, T. Determination by LC-MS of Juvenile Hormone Titers in Hemolymph of the Silkworm, Bombyx mori. J. Agric. Chem. Soc. Jpn. 2013, 77, 988-991. [CrossRef]

35. Wang, L.; Feng, Z.; Wang, X.; Wang, X.; Zhang, X. DEGseq: An R package for identifying differentially expressed genes from RNA-seq data. Bioinformatics 2010, 26, 136-138. [CrossRef]

36. Livak, K.J.; Schmittgen, T. Analysis of relative gene expression data using real-time quantitative PCR and the 2-DDCt method Methods 2001, 25, 402-408. [CrossRef] [PubMed]

37. Shpigler, H.Y.; Magory Cohen, T.; Ben-Shimol, E.; Ben-Betzalel, R.; Levin, E. Juvenile hormone functions as a metabolic rate accelerator in bumble bees (Bombus terrestris). Horm. Behav. 2021, 136, 105073. [CrossRef]

38. Hartfelder, K.; Engels, W. Social Insect Polymorphism: Hormonal Regulation of Plasticity in Development and Reproduction in the Honeybee. Curr. Top. Dev. Biol. 1998, 40, 45-77. [CrossRef] 
39. Barchuk, A.R.; Cristino, A.S.; Kucharski, R.; Costa, L.F.; Simoes, Z.L.; Maleszka, R. Molecular determinants of caste differentiation in the highly eusocial honeybee Apis mellifera. BMC Dev. Biol. 2007, 7, 70. [CrossRef] [PubMed]

40. Li, W.; Huang, Z.Y.; Liu, F.; Li, Z.; Yan, L.; Zhang, S.; Chen, S.; Zhong, B.; Su, S. Molecular cloning and characterization of juvenile hormone acid methyltransferase in the honey bee, Apis mellifera, and its differential expression during caste differentiation. PLoS ONE 2013, 8, e68544. [CrossRef] [PubMed]

41. Dominguez, C.V.; Maestro, J.L. Expression of juvenile hormone acid O-methyltransferase and juvenile hormone synthesis in Blattella germanica. Insect Sci. 2018, 25, 787-796. [CrossRef] [PubMed]

42. Xie, X.; Tao, T.; Liu, M.; Zhou, Y.; Liu, Z.; Zhu, D. The potential role of juvenile hormone acid methyltransferase in methyl farnesoate (MF) biosynthesis in the swimming crab, Portunus trituberculatus. Anim. Reprod. Sci. 2016, 168, 40-49. [CrossRef] [PubMed]

43. Marchal, E.; Zhang, J.; Badisco, L.; Verlinden, H.; Hult, E.F.; van Wielendaele, P.; Yagi, K.J.; Tobe, S.S.; Vanden Broeck, J. Final steps in juvenile hormone biosynthesis in the desert locust, Schistocerca gregaria. Insect Biochem. Mol. Biol. 2011, 41, 219-227. [CrossRef] [PubMed]

44. Tu, M.P.; Yin, C.M.; Tatar, M. Mutations in insulin signaling pathway alter juvenile hormone synthesis in Drosophila melanogaster. Gen. Comp. Endocrinol. 2005, 142, 347-356. [CrossRef]

45. Huang, J.; Marchal, E.; Hult, E.F.; Zels, S.; Vanden Broeck, J.; Tobe, S.S. Mode of action of allatostatins in the regulation of juvenile hormone biosynthesis in the cockroach, Diploptera punctata. Insect Biochem. Mol. Biol. 2014, 54, 61-68. [CrossRef]

46. Mamidala, P.; Wijeratne, A.J.; Wijeratne, S.; Kornacker, K.; Sudhamalla, B.; Rivera-Vega, L.J.; Hoelmer, A.; Meulia, T.; Jones, S.C.; Mittapalli, O.J.B.G. RNA-Seq and molecular docking reveal multi-level pesticide resistance in the bed bug. BMC Genom. 2012, 13, 6. [CrossRef]

47. Mao, W.; Berenbaum, S. CYP9Q-mediated detoxification of acaricides in the honey bee (Apis mellifera). Proc. Natl. Acad. Sci. USA. 2011, 108, 12657-12662. [CrossRef] [PubMed]

48. Wang, K.; Fan, R.L.; Ji, W.N.; Zhang, W.W.; Chen, X.M.; Wang, S.; Yin, L.; Gao, F.C.; Chen, G.H.; Ji, T. Transcriptome Analysis of Newly Emerged Honeybees Exposure to Sublethal Carbendazim During Larval Stage. Front. Genet. 2018, 9, 426. [CrossRef]

49. Li, H.; Liu, S.; Chen, L.; Luo, J.; Zeng, D.; Li, X. Juvenile hormone and transcriptional changes in honey bee worker larvae when exposed to sublethal concentrations of thiamethoxam. Ecotoxicol. Environ. Saf. 2021, 225, 112744. [CrossRef] [PubMed]

50. Sutherland, T.D.; Unnithan, G.C. A cytochrome P450 terpenoid hydroxylase linked to the suppression of insect juvenile hormone. Proc. Natl. Acad. Sci. USA 1998, 95, 12884. [CrossRef] [PubMed]

51. Yaguchi, H.; Masuoka, Y.; Inoue, T.; Maekawa, K. Expressions of juvenile hormone biosynthetic genes during presoldier differentiation in the incipient colony of Zootermopsis nevadensis (Isoptera: Archotermopsidae). Appl. Entomol. Zool. 2015, 50, 497-508. [CrossRef] 